\title{
The stability of bag dust collection system
}

\author{
Yumeng Cai
}

\author{
School of North China Electric Power University, Baoding 071003, China \\ 2469103191@qq.com
}

\begin{abstract}
Keywords: Bag filter, Analytic Hierarchy Process, Fuzzy evaluation method, Grey prediction method
\end{abstract}

\begin{abstract}
This paper studies the stability of operation of waste incineration power plant dust bag system, the first to adopt comprehensive evaluation method based on fuzzy analytic hierarchy process to build dust removal system stability model, the stars finally got nine factors affect the stability of the system accounted for weight, obtained by fitting a linear function of the stability evaluation reference value, in order to evaluate the stability of the dust removal system. Then apply a gray prediction model has been considered in the total area under the premise of stable pollutant emissions a year, combined with a given amount of emissions per unit area calculated ambient allowed limit. Based on the above analysis of the stability of the final EPA proposed a number of comprehensive environmental monitoring program and recommend that it be based on our evaluation of the stability of a system in order to take the appropriate time to monitor changes in the focus and monitoring, as EPA provided some data analysis thereby facilitating its monitoring.
\end{abstract}

\section{Introduction}

Today, it is the only way to maintain the sustainable development of our society by incineration. Therefore, the quantitative analysis on stability of bag filter, can not only reveal the defects of flue gas waste incineration technology in order to promote the dedusting technology progress, but also to optimize the burning conditions control and operation and maintenance regulations for help. Therefore, comprehensive study of the current waste incineration effect of bag type dust collector system of power plant soot emissions of various factors, constructing mathematical model for stability analysis of bag type dust removal system problems, and analyze the effect of operation on the stability of surrounding environment smoke emissions.

\section{A model of influencing factors of dust removal system stability based on AHP}

\section{Establish a dedusting system hierarchy.}

When applying $\mathrm{AHP}^{[1]}$ decision analysis problem, we must first issue structured, hierarchy, construct a hierarchical structure model. In this model, complex problem is decomposed into elemental components. These elements and their attributes and the relationship between the formation of a number of levels. Previous level the elements as a guideline for the next level of the relevant elements from the dominant role. These levels can be divided into three categories:

( $\mathrm{i}$ ) the highest level: This level is only one element, it is generally intended target or desired results analysis of the problem, also known as the target layer.

( II ) intermediate layer: This level contains the goals involved in the intermediate links, which may consist of a number of levels, including the guidelines need to be considered, the sub-criteria, also known as the criterion level.

(III) the lowest level: this level includes alternative for achieving the objectives of the various measures, the decision-making programs, also known as layer measures or programs layers.

First nine indicators divided into three layers, namely the target layer, criteria layer, project layer, as shown below: 


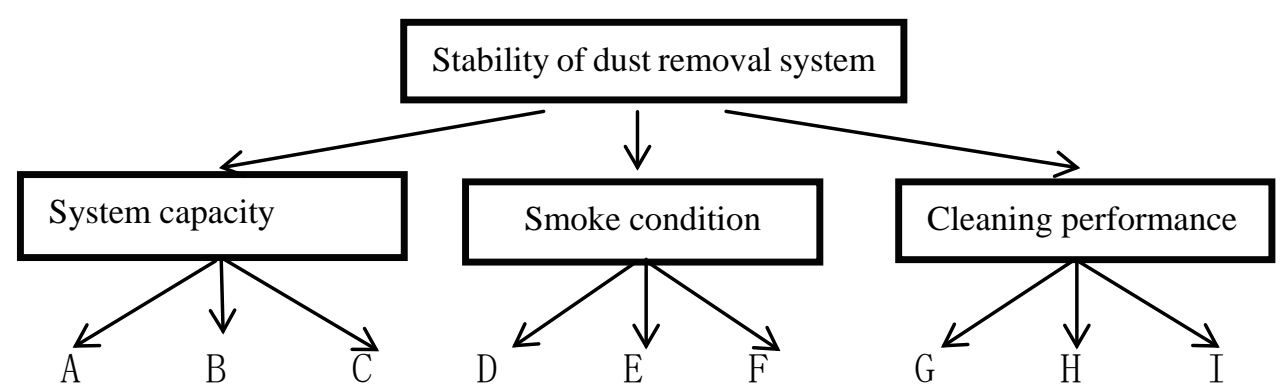

\section{Judgment matrix.}

First analyze the impact on the target layer, criteria layer, the nine indicators divided into three categories, it has three elements, namely the ability to dedusting system, flue gas, the cleaning performance, with representation and the upper layer affect the target ratio. After analysis of data, it can be approximated by the following factors:

Matrix A is obtained.

$$
\mathrm{a}_{12}=5: 3, \mathrm{a}_{12}=5: 1, \mathrm{a}_{23}=3: 1
$$

\section{Single-level sorting and consistency check.}

Using matlab language, of a matrix A maximum eigenvalue, $\lambda=2.9999$, positive reciprocal matrix consistency test, comparing the consistency index table available:

$$
\mathrm{CR}=0<0.42
$$

So through the consistency check.

\section{The normalized get weight vector.}

Through MATLAB to normalized, eigenvectors are the standardization, w= $(0.5555,0.3333 .0 .1111)$, the system capacity, dust and ash proportion to the stability of systems (0.5555,0.3333.0.1111).

\section{Calculating the weight vector.}

Construction project level guidelines guidelines for each positive reciprocal matrix. Similarly to the above matrix consistency processing, pass the test.

\section{Based on fuzzy evaluation method to establish the stability evaluation model of dust removal system}

The establishment of fuzzy comprehensive evaluation model ${ }^{[2]}$ mainly includes the following steps:

(1) Determining factor set. All these factors constitute a set of evaluation index system, that is, the factor set:

$$
\mathrm{U}=\{\mathrm{u} 1 \ldots \mathrm{Un}\}
$$

(2) Determining the set of comments. As a result of the evaluation value of each index is different, often will form different, such as the evaluation of the performance of a good, good, medium, poor, poor, etc.. A collection made up of various decisions is a collection of comments.

$$
\mathrm{V}=\{\mathrm{v} 1 \ldots \mathrm{Vn}\}
$$

(3) Determine the weight of each factor. In general, these factors have concentrated in the comprehensive assessment of the role is not the same, the results of comprehensive evaluation and evaluation not only various factors, but also depends on the various factors on the comprehensive evaluation of the role to a large extent, it is necessary to determine the one because of weight distribution between. It is a fuzzy vector $U$, denoted as A.

(4) Determine the fuzzy comprehensive judgment matrix. For the index, the membership of each comment is a fuzzy subset on $\mathrm{V}$.

The fuzzy judgment matrix of each index is $R$, which is a fuzzy relation matrix from $U$ to $V$.

(5) Comprehensive evaluation. If there is a fuzzy relation $\mathrm{V}$ from $\mathrm{U}$ to $\mathrm{R}$, then $\mathrm{R}$ can be used to obtain a fuzzy transformation

$$
\text { TR:F (U) - F (V) }
$$


Therefore, the comprehensive evaluation result can be obtained by $\mathrm{B}=\mathrm{AR}$.

The comprehensive evaluation can be regarded as a fuzzy vector on $\mathrm{V}$, denoted as $\mathrm{B}$.

\section{Grey prediction model of regional emission}

After checking the information obtained as shown in the annual pollutant emissions data BTH Region $^{[3]}$ in the Table 1:

Table 1 Beijing, Tianjin and Hebei region industrial dust emissions (10000 tons)

\begin{tabular}{c|c|c}
\hline numbers & years & L \\
\hline 1 & 2009 & 96.6 \\
\hline 2 & 2010 & 95.8 \\
\hline 3 & 2011 & 102.7 \\
\hline 4 & 2012 & 114.6 \\
\hline 5 & 2013 & 127.7 \\
\hline 6 & 2014 & 158.6 \\
\hline
\end{tabular}

Based on the fuzzy comprehensive evaluation method of the weight of each factor score obtained by analysis of reference value of stability formula $y$, by data simulation and soot emissions corresponding to the six years of stability of reference values, as shown in the Table 2:

Table 2 the value of $y$

\begin{tabular}{c|c|c}
\hline numbers & years & $\mathrm{y}$ \\
\hline 1 & 2009 & 5.68 \\
\hline 2 & 2010 & 5.70 \\
\hline 3 & 2011 & 6.08 \\
\hline 4 & 2012 & 6.37 \\
\hline 5 & 2013 & 8.51 \\
\hline 6 & 2014 & 9.32 \\
\hline
\end{tabular}

The main steps of Grey prediction model are as follows:

(1)inspection and processing of data

First of all, in order to ensure the feasibility of the modeling method, it is necessary to do the necessary inspection and processing to the known data column, and calculate the order of the sequence

(2) build model

According to the grey prediction model GM $(1,1)$, the predicted value can be obtained

(3) test forecast value

(I):the residual value of the residual test $(\mathrm{k})$, if epsilon epsilon $(\mathrm{k})<0.2$, will be considered to general requirements; if epsilon $(\mathrm{k})<0.1$, will be considered to achieve higher requirements.

(II) level than the deviation test: first, from the reference data to calculate the level ratio, and calculated the corresponding coefficient a development level than if the deviation $\mathrm{P}(\mathrm{k})<0.2$, that can reach the general requirements; if $\mathrm{P}(\mathrm{k})<0.1$, will be considered to achieve higher requirements.

(4) forecast

By the model GM $(1,1)$, the predicted value of the designated urban area, according to the actual needs of the problem, given the corresponding forecast. 


\section{Proposal for comprehensive monitoring of environmental protection}

Based on the above, such as AHP, fuzzy comprehensive evaluation method, grey prediction method for the stability analysis of influence factors of modeling, visible to ensure stable efficient operation of the system is the key, according to the mathematical model given the following comprehensive environmental monitoring program established and suggestions:

(1) environmental protection departments set up monitoring index is mainly reflected in the monitoring of toxic substances, including dioxins, dust, hydrogen sulfide and other toxic substances emissions, its reason lies in: on the one hand, based on the health consciousness, people of dioxin, dust, hydrogen sulfide and other toxic substances control requirements and emission control the amount is more sensitive; on the other hand, the emission of toxic substances is an important factor affecting the stability of bag type dust removal system.

(2) monitoring the time and place of the environmental protection departments set up mainly focus on the stability of the system after a moment's stability is poor evaluation of the mathematical model, the reason is: on the one hand, the current waste incineration defect sustained and stable operation of dust removal process can't, cause the public to cause harm doubts about waste incineration; on the other on the one hand, when the poor stability of bag type dust removal system, fault discharge time may exist longer, resulting in even the incineration plant emissions do not exceed the standard, but there are still problems limit the total emissions exceed the standard, will bring great influence to the local environment deterioration.

(3) The monitoring results of the environmental protection department shall be submitted in time and accurately analyzed and evaluated.

\section{Conclusions}

This paper uses AHP, fuzzy comprehensive evaluation method, grey prediction method for the stability analysis of influence factors of modeling, visible to ensure stable efficient operation of the system is the key, and the bag filter velocity, resistance coefficient, bag, temperature, the content of toxic substances, particle diameter, pulse period, pulse time, pressure and so on an important factor in the stability of the system. Through the establishment of mathematical model, quantitative analysis of stability of bag filter, can not only reveal the defects of flue gas waste incineration technology in order to promote the dedusting technology progress, but also can to optimize the burning conditions control and operation and maintenance regulations for help, and according to the established mathematical model to the Environmental Protection Agency proposed comprehensive environmental monitoring scheme and corresponding suggestions.

\section{References}

[1] Shoukui Si,Xijing Sun.Mathematical Modeling Algorithms and Applications.National Defence Industry Press.2015.

[2] Lijun Zhang,Juan Yang.Fuzzy comprehensive evaluation of scientific and technological achievements based on expert weight[J].Technology and Economy.,2011,v.24;No.14204:1-5.

[3] Ying Xi. Mechanism and influencing factors of bag filter[J].Special Purpose Rubber Products,2003,01:29-33. 